\title{
Two- versus three-dimensional ultrasound in the second and third trimester of pregnancy: impact on recognition and maternal-fetal bonding. A prospective pilot study
}

\author{
Olav Lapaire $\cdot$ Judith Alder $\cdot$ Regina Peukert $\cdot$ \\ Wolfgang Holzgreve $\cdot$ Sevgi Tercanli
}

Received: 12 December 2006 / Accepted: 27 March 2007 / Published online: 25 April 2007

(C) Springer-Verlag 2007

\begin{abstract}
Objective To assess the impact of three-dimensional (3D) versus two-dimensional (2D) ultrasound (US) on maternalfetal bonding.

Study design Prospective randomized pilot study among low risk women with singleton fetuses in the second and third trimester. Dependent on the randomization pattern, US was commenced either with 2D US or 3D US and the effects were recorded with standardized questionnaires.

Results Sixty patients were included. Although the quality of 2D US, assessed by the examinator, was superior to 3D US, maternal recognition was higher with 3-D US $(P=0.004)$. With 2D US, nulliparous patients had significantly more difficulties visualizing the fetus, than multiparous $(P=0.03)$. However, the maternal preference of $3 \mathrm{D}$ US had no significant impact on maternal-fetal bonding.

Conclusion Ultrasound had no significant effect on maternal-fetal bonding. Three-dimensional images may facilitate recognition of the fetus, but 3D US did not have higher impact on maternal-fetal bonding. This finding may be a reason not to consider $3 \mathrm{D}$ ultrasound for routine scanning.
\end{abstract}

O. Lapaire $(\bowtie) \cdot$ J. Alder $\cdot$ R. Peukert $\cdot$ W. Holzgreve $\cdot$ S. Tercanli Department of Obstetrics and Gynecology,

University Hospital Basel, Spitalstrasse 21,

CH-4031 Basel, Switzerland

e-mail: olapaire@uhbs.ch

J. Alder

e-mail: jalder@uhbs.ch

W. Holzgreve

e-mail:wholzgreve@uhbs.ch

S. Tercanli

e-mail: stercanli@uhbs.ch
Keywords Ultrasonography · Prenatal · Maternal-fetal relations · Prenatal care

\section{Introduction}

The continual technical advances made in the field of prenatal ultrasound (US) have rapidly improved diagnostic and therapeutic capabilities. Although current standard of care is almost exclusively to perform two-dimensional (2D) ultrasound, three-dimensional (3D) and recently four dimensional (4D) ultrasound have found its way into clinical practice over the last 10 years. In the future, additional clinical indications may be specifically identified for 3D US due to its ability to evaluate anatomical structures and pathologic findings with multiplanar and surface-rendered images. However, the current demands, especially from patient's side, have lead to a rapidly increasing number of 3D examinations being performed, many of them either without a well-defined indication or not within a circumscribed trial-setting.

While positive short-term consequences of 2D ultrasound on pregnant women's well-being are well established, specific effects of 3D sonography on maternal-fetal bonding, recognition of the fetus, and effects on the partnership have not been well investigated [1,2]. Under optimal conditions, 3D US provides high quality images that may be easier to perceive than those generated by $2 \mathrm{D}$ examinations. Therefore, recognition of the fetus, as well as maternal-fetal bonding, may be better facilitated with 3D images. However, suboptimal conditions may lead to disappointment because the results may not fit with the mother's high expectations and envisaged images of the growing fetus.

We therefore conducted this pilot study to determine whether the addition of $3 \mathrm{D}$ to $2 \mathrm{D}$ US in the second/third 
trimester of pregnancy facilitates maternal recognition and has an impact on maternal-fetal bonding, taking into consideration the parity of the patient. The operator's satisfaction with the achieved quality of the images and the success rate of $2 \mathrm{D}$ and $3 \mathrm{D}$ ultrasound were also analyzed.

\section{Patients and methods}

Study design and sample description

This prospective randomized pilot study was performed between September 2000 and August 2002. Sixty healthy German speaking pregnant women with unremarkable medical history and singleton pregnancies were asked during the routine prenatal visits to participate after reading a brief information sheet and signing informed consent. If present, their partners were also included. Exclusion criteria were higher-order gestations, intrauterine growth retardation, malformations and aneuploidies in the ongoing pregnancy, stillbirth and other abnormalities in prior pregnancies, oligohydramnios and a body mass index $>35$. The patients were randomly assigned via a computer-generated numbered list to one of two groups: either to receive first a 2D US, followed by a 3D US (Group 1), or to receive first a 3D US, followed by a 2D US (Group 2). These sequences were chosen in order to control for a possible bias in patient's evaluation resulting from the specific order of the scans.

One trained operator (RP) performed all ultrasound examinations in the ultrasound department of the University Hospital Basel, Switzerland, between the 23rd and 34th week of gestation. All prenatal US screenings in the first and second trimester had been normal. The parents-to-be observed the fetus on a separate screen.

\section{Ultrasound procedure}

The scans included a general overview of the intrauterine pregnancy, followed by measurements of the biparietal and frontooccipital diameter, abdominal circumference and femur length. The placenta was scanned and the amniotic fluid volume was estimated. In a second step, the operator aimed to specifically visualize fetal structures such as the face, which may evoke maternal or paternal emotions. Each examination took the same amount of time (approx. $30 \mathrm{~min}$ ) and was accompanied by simultaneous explanations. All examinations were performed in the same room, in a quiet and relaxed atmosphere. The scans were performed with the Voluson 530D system (GE Medical Systems, Kretz Ultrasound, Zipf, Austria), which includes a 3D abdominal probe (S-vSW 3-5). All documentations were stored in the PIA fetal database (GE Medical Systems). The image acquisition time of the 3D pictures varied between approximately $5-15 \mathrm{~s}$, depending on the preselected size of the volume box, which can be adjusted individually. The image volume generated by the system was displayed on the screen with three orthogonal scan planes in a perpendicular position. By rotating the volume body within the three axes and by shifting the centre of rotation along these axes, different parts of the presented volume could be generated. A further processing of the data set could be achieved by the software option "interactive volume rendering". This option generates high quality images of the surface and transparent images of the examined region. Undesired regions were cut off for increased performance-enhancement. After each examination, the operator rated the quality of the performed ultrasound according to a graded score system ( $1=$ best quality, $5=$ poorest quality $)$ and with a classification into three major groups: successful display, moderate display, no display.

\section{Patient's questionnaire}

After each examination, the patient's and if present, her partner's experiences during the scan were assessed by structured questionnaires (see appendix), without the presence of the operator, which could have influenced their rating. At the end of both cycles an additional form was completed, concerning the preferences of $2 \mathrm{D}$ or $3 \mathrm{D}$ and the reasons leading to this decision. Due to the lack of a German version of the "Cranley maternal-fetal attachment scale" or a similar instrument, questionnaires were developed specifically for this study, incorporating the knowledge of a psychologist (JA) and experts in prenatal ultrasound (RP, ST) [3]. In total, five questions focused on the recognition of the fetus and the assessment of $2 \mathrm{D} / 3 \mathrm{D}$ ultrasound. Additional questions focused on maternal-fetal relationship, partner's opinion, feeling of closeness towards the partner and satisfaction with the examination. All questions, except those concerning the preferences of $2 \mathrm{D}$ or $3 \mathrm{D}$ US and the reasons leading to this decision, were answered on a 5-point Likert scale (e.g. 1 corresponding to: "agree completely"; 5 corresponding to: "not at all"). The additional questions concerning preferences of ultrasound modes were dichotomous. One additional question offered the possibility of an open answer (Fig. 1).

\section{Statistical analysis}

Questionnaire data was normally distributed (KolmogorovSmirnov test). $P$ values were two tailed and the level of statistical significance was 0.05 . The dependent variables (maternal recognition, bonding, feeling of closeness to the partner and satisfaction with the examination) were analyzed using a general linear model for repeated measures, 


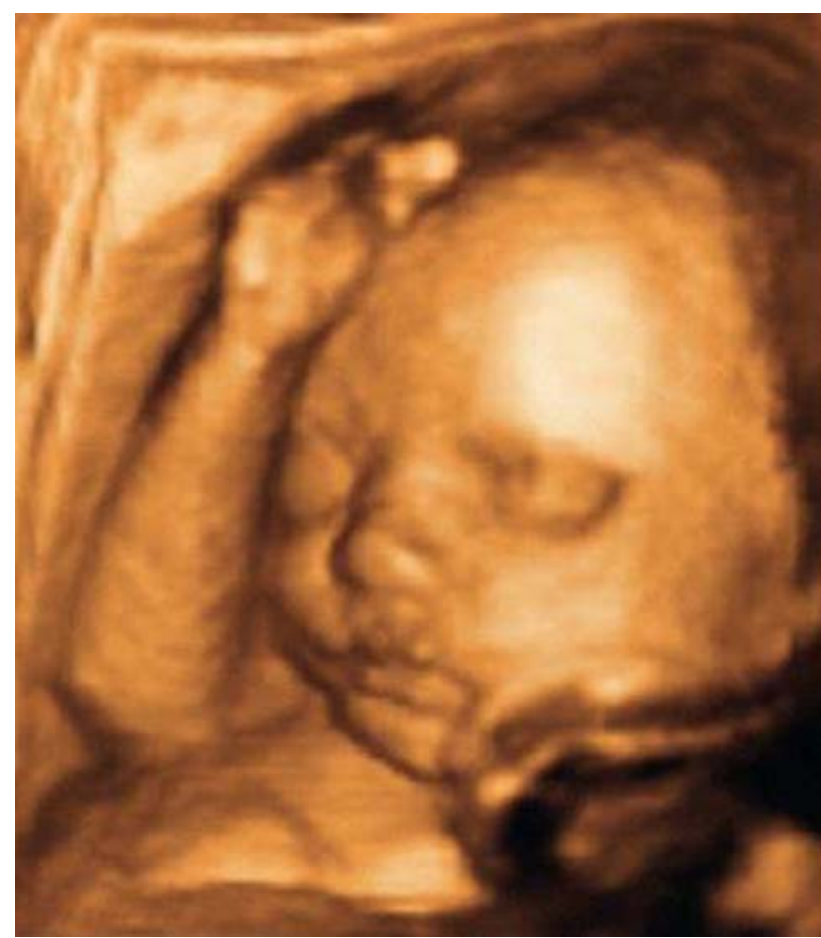

Fig. 1 Three-dimensional image of a fetal head in the 27th week of gestation (performed with the Voluson 530D system (GE Medical Systems, Kretz Ultrasound, Zipf, Austria), which includes a 3D abdominal probe (S-vSW 3-5)

since the same subjects were viewing $2 \mathrm{D} / 3 \mathrm{D}$ images, with the parity of the patient taken into consideration.

Results are reported as mean \pm standard deviation (SD) and percentages. All analyses were performed using SPSS 13.0 (SPSS Inc., Chicago, USA).

\section{Results}

There were no statistically significant differences in the clinical characteristics or questionnaire answers of the 60 women enrolled in both groups, which were defined by the sequence of ultrasound examinations. Mean gestational age was 27.4 (SD 2.3), 55\% of the participants were nulliparous, and the mean parity was 0.6 (SD 0.8 ). Table 1 illustrates descriptive data for the entire group relating to $2 \mathrm{D}$ and 3D scans. Comparing both modes of ultrasound, the recorded quality was slightly higher in $2 \mathrm{D}$ examinations (42/60 examinations: successful, 18/60: moderate display), compared to 3D examinations (41/60 examinations: successful, 16/60: moderate display, 3/60 no display).

Impact of dimensionality of scans

Recognition of 3D images was significantly associated with examiner's reporting on the quality of the scan $(r=0.46$,
Table 1 Characteristics of both scan modalities

\begin{tabular}{lll}
\hline & $2 \mathrm{D}$ & $3 \mathrm{D}$ \\
\hline Recognition of fetus $^{\mathrm{a}}$ & $1.9(0.9)$ & $1.7(0.9)$ \\
Relationship towards fetus $^{\mathrm{b}}$ & $4.4(0.7)$ & $4.3(0.9)$ \\
Partner's opinion $^{\mathrm{b}}$ & $4.2(0.9)$ & $4.2(0.9)$ \\
Feeling of closeness towards the partner $^{\mathrm{a}}$ & $1.5(0.7)$ & $1.6(0.8)$ \\
Satisfaction with examination $^{\mathrm{a}}$ & $1.3(0.6)$ & $1.3(0.6)$ \\
Examiner's rating on image quality & & \\
Positive $_{\text {Fair }}$ & $70 \%$ & $68.3 \%$ \\
Poor & $30 \%$ & $26.7 \%$ \\
\hline
\end{tabular}

Mean scores of questionnaire answers and examiner ratings for $2 \mathrm{D}$ and 3D scans $(N=60)$

a 1 : highest rating, 5 : lowest rating

b 5 : highest rating, 1: lowest rating

$P<0.01)$ while there was no such association for 2 D scans. Nevertheless, $87.5 \%$ of the women preferred 3D scans, while only $12.5 \%$ preferred $2 \mathrm{D}$ scans.

There were no differences in the dependent variables with respect to group adherence (sequence of scans), therefore we did not control further for this factor. General linear model repeated measures for intra-subject differences were calculated to compare answers in the dependent variables on $2 \mathrm{D}$ versus $3 \mathrm{D}$ controlling for parity. Recognition of $3 \mathrm{D}$ scans was significantly better, indicated by lower mean scores $(F=13.53, P<0.01)$ with a significant interaction with parity (scan x parity, $F=10.38, P<0.01$ ) (Table 2). This was due to impaired recognition of $2 \mathrm{D}$ scans in nulliparae compared to women who had already given birth (T $=2.10, P<0.05)$. Overall, only $12 \%(7 / 60)$ of the patients stated that 2D US images were more easily recognizable and $6 \%(4 / 60)$ of the patients found no difference between the two modes.

Dimensionality of the scan did not have an effect on maternal bonding $(F=0.06, P<0.9)$, paternal opinion $(F=0.33, p<0.6)$ or satisfaction with the examination $(\mathrm{F}=1.11, P<0.3)$.

Table 2 Maternal and partners effects after 2D and 3D imaging of the fetus

\begin{tabular}{llll}
\hline & $3 \mathrm{D}$ & 2D & $P^{\mathrm{a}}$ \\
\hline Maternal recognition & $1.7(0.9)$ & $1.9(0.9)$ & $<0.01$ \\
Maternal-fetal bonding & $4.3(0.9)$ & $4.4(0.7)$ & NS \\
Partner's attraction to the scan & $4.2(1.0)$ & $4.2(0.9)$ & NS \\
Satisfaction with examination & $1.3(0.6)$ & $1.3(0.6)$ & NS
\end{tabular}

Impact of dimensionality of scan on questionnaire scores (mean and standard deviation)

a General linear model repeated measures for intra subject differences to compare answers on $2 \mathrm{D}$ versus $3 \mathrm{D}$ controlling for parity 


\section{Discussion}

Ultrasound as a diagnostic and therapeutic tool has been increasingly utilized in prenatal care. During recent years, the technique of 3D US has become widely available in clinical practice, in addition to conventional 2D US, and has gained an extraordinary popularity, in part because of the more realistic 3D rendering of fetal structures such as the fetal face and limbs and partly due to certain improvements in showing specific fetal malformations of the body surface, spine, cranium and face $[4,5]$. However, suboptimal recorded 3D images may also lead to disappointment, because they do not meet with maternal expectations.

With this study we sought to evaluate the impact of $2 \mathrm{D}$ and 3D images on maternal-fetal bonding and fetal recognition in the second and third trimester of pregnancy. We found no differences between 2D and 3D US in short term effects with regard to maternal-fetal bonding. The explanation may be that there is a pre-existing high level of attachment in the late second and third trimester, indicating that the majority of the maternal-fetal bonding process has already been established earlier in pregnancy. The high levels of attachment at this stage may have been influenced by fetal movements [6]. However, in our study we did not control for preexistent maternal-fetal attachment. Therefore, the conclusion is limited to a comparison of $2 \mathrm{D}$ versus $3 \mathrm{D}$ US. Furthermore, the levels of attachment were not influenced by any concomitant negative factors in this study (e.g. malformations in previous scans, undesired pregnancies, burden of single parent households), as these had been initially screened out. Nevertheless, it has been suggested that the visualization of the growing fetus may activate additional emotions, which further trigger sentiments of prenatal attachment [7]. Eighty-two percent of the patients, irrespective of the order of the examinations, reported a higher preference for 3D pictures, either due to closeness to reality or better perceptibility. However, the mode of ultrasound (2D/3D) had no impact on the reported maternalfetal relationship, on the expressed feelings towards the partner or on the reported satisfaction with the performed examinations.

The higher rate of recognizability of the fetus with 3D images is in agreement with previously published studies $[8,9]$. Furthermore, a recently published study reported a positive influence of $3 \mathrm{D}$ ultrasound with regard to maternal perception of their fetuses [1]. Additionally, the mothers showed their 3D ultrasound pictures to a greater number of acquaintances than 2D images. However, data was collected retrospectively by telephone follow-up surveys of a mixed group with high-risk and low-risk pregnancies between 1 and 24 months after birth. The latest improvements in image resolution, as well as the implementation of 4D in daily practice, may have a further significant influence in maternal-fetal bonding. Additional studies are needed to investigate both issues.

In our study, the patients' visual impressions of the 3D examinations was in line with the examinator's opinion on the quality of the pictures. In contrast, differences in quality levels of 2D images did not have an influence on patient's visualization, probably due to generally lower levels of recognition.

The lack of familiarity with the method and images may make it difficult to interpret 2D images, especially for nulliparae. The high resolution of the latest 3D machines simplifies the interpretation by creating life-like images of the fetuses. However, the visual impression decreases with lower quality due to defects in image construction, unfavorable position of the targeted area, or lack of amniotic fluid.

T. Chudleigh stated that "scanning for parental pleasure, i.e. bonding, should be considered as a part of the obstetrical ultrasound" [10]. This statement has been incorporated into our daily work and into standard ultrasound examinations, and this has been shown in diverse clinical trials to decrease the anxiety of pregnant women and to increase maternal-fetal bonding $[11,12,13,14]$. In contrast to many studies investigating the impact of $2 \mathrm{D}$ ultrasound on maternal-fetal bonding, only very little data are available for 3D sonography $[1,2]$. With the increasing number of performed 3D scans, its psychological influence needs to be further investigated. Only a few studies focus on the partner's experience with regard to prenatal ultrasound. Those few existing studies are in agreement with our data, describing no significant differences in paternal compared to maternal behavior $[15,16]$. A potential weakness of our study was that we did not use a validated questionnaire, as no German questionnaire was available. Additionally, there may be a selection bias because of the inclusion and exclusion criteria, and because of the voluntary nature of the study. A power calculation at the beginning of the study planning was made on the basis of hypothetical considerations, rather than on robust data assessed by previous studies. Furthermore, the relatively wide range of gestational age may have prevented specific observation of gestational age-dependent effects. However, none of these factors seem likely to have a significant impact on the main results, in particular since our primary focus was to investigate whether the addition of 3D to 2D ultrasound in the second/ third trimester of pregnancy facilitates maternal recognition and has a significant impact on maternal-fetal bonding.

In conclusion, we did not find a difference between 2D and 3D ultrasound in maternal-fetal bonding in the second half of pregnancy. Nevertheless the data demonstrated that there exists a higher maternal recognition of the fetus in 3D images. A larger study could be performed to investigate the point in time in which US $(2 \mathrm{D}, 3 \mathrm{D}$, or possibly even 4D) may have a significant impact on maternal-fetal bond- 
ing. At the moment, however, based on our findings, 3D ultrasound should not be advocated yet as a standard screening tool. The use of 3D ultrasound is recommended only when medically indicated reasons exist, due to the increasing costs of health care, which can at least partially be attributed to patients' ascending expectations.

\section{Appendix}

Translated questionnaires, used in the study: Questionnaire 1 (after the first examination)

- How well did you recognize your baby in the ultrasound?

- How would you assess your relationship to the baby at this moment (after the examination)?

- How did the images appeal to your partner?

- How close do you feel to your partner?

- How satisfied are you with the examination?

Questionnaire 2 (after the second examination)

- How well did you recognize your baby in the ultrasound?

- How would you assess your relationship to the baby at this moment (after the examination)?

- How did the images appeal to your partner?

- How close do you feel to your partner?

- How satisfied are you with the examination?

Why did you find the 2D images to be better?

- Better recognizable

- Closer to reality

- Other reasons

Why did you find the 3D images to be better?

- Better recognizable

- Closer to reality

- Other reasons

Which image do you prefer?

- 2D ( ) 3D ( ).

- Compared to the other image, how much better did you find it to be?

\section{References}

1. Ji EK, Pretorius DH, Newton R, Uyan K, Hull AD, Hollenbach K, Nelson TR (2005) Effects of ultrasound on maternal-fetal bonding: a comparison of two- and three dimensional imaging. Ultrasound Obstet Gynecol 25:473-477

2. Pretorius DH, Uyan KM, Newton R, Hull A, James G, Nelson T (2001) Effects of US on maternal-fetal bonding: 2D versus 3D. Ultrasound Obstet Gynecol 18(Suppl 1):20 (Abstract F35)

3. Cranley MS (1981) Development of a tool for the measurement of maternal attachment during pregnancy. Nurs Res 30:281-284

4. Timor-Tritsch IE, Platt LD (2002) Three-dimensional ultrasound experiences in obstetrics. Curr Opin Obstet Gynecol 14:569-575

5. Dyson RL, Pretorius DH, Budorick NE, Johnson DD, Sklansky MS, Cantrell CJ, Lai S, Nelson TR (2000) Three-dimensional ultrasound in the evaluation of fetal anomalies. Ultrasound Obstet Gynecol 16:321-328

6. Heidrich SM, Cranley MS (1988) Effect of fetal movement, ultrasound scans, and amniocentesis on maternal-fetal attachment. Nurs Res 38: 81-84

7. Siddiqui A, Hagglof B (2000) Does maternal prenatal attachment predict postnatal mother-infant interaction?. Early Hum Dev 59:13-25

8. Pretorius DH, Nelson TR (1995) Fetal face visualization using three dimensional ultrasonography. J Ultrasound Med 14:349-356

9. Nelson TR, Elvins TT (1993) Visualization of 3D ultrasound data. IEEE Comput Graph Appl 13:50-57

10. Chudleigh T (1999) Scanning for pleasure. Ultrasound Obstet Gynecol 14:369-371

11. Brown GF (1988) Short-term impact of fetal imaging on prenatal stress and anxiety. Pre Peri Nat Psychol 3:25-40

12. Zlotogorski Z, Tadmor O, Duniec E, Rabinowitz R, Diamant Y (1995) The effect of the amount of feedback on anxiety levels during ultrasound scanning. J Clin Ultrasound 24:21-24

13. Langer M, Ringler M, Reinold E (1988) Psychological effects of ultrasound examinations: changes of body perception and child image in pregnancy. J Psychosomatic Obstet Gynecol 8:199-208

14. Eurenius K, Axelsson O, Gällstedt-Fransson I, Sjöden PO (1997) Perception of information, expectations and experiences among women and their partners attending a second-trimester routine ultrasound scan. Ultrasound Obstet Gynecol 9:86-90

15. Villeneuve C, Laroche C, Lippman A, Marrache M (1988) Psychological aspects of ultrasound imaging during pregnancy. Can J Psychiatry 33:530-538

16. Eurenius K, Axelsson O, Gällstedt-Fransson I, Sjöden PO (1997) Perception of information, expectations and experience among women and their partners attending a second-trimester routine ultrasound scan. Ultrasound Obstet Gynecol 9:86-90 